\title{
Primary spontaneous pneumothorax in a recreational athlete
}

\author{
F J van der Colff, ${ }^{1,2} \mathrm{MB} \mathrm{ChB}$; D C Janse van Rensburg, ${ }^{2,3} \mathrm{MD}$ \\ ${ }^{1}$ Private Practitioner, Potchefstroom, South Africa \\ ${ }^{2}$ Section Sports Medicine, Faculty of Health Sciences, University of Pretoria, South Africa \\ ${ }^{3}$ Exercise SMART Team, University of Pretoria, South Africa
}

Corresponding author: F J van der Colff (medfvandercolff@yahoo.com)

\begin{abstract}
Primary spontaneous pneumothorax (PSP) is relatively uncommon in the athletic population. Because of the subtle nature of the symptoms, the diagnosis is easily missed, which can lead to unnecessary prolonged discomfort and recovery time for the athlete. There is currently a lack of evidence in the literature concerning treatment and return-to-play protocols referring specifically to PSP within the athletic community. This case report highlights the predisposing and important factors in the history of a 34-year-old recreational male athlete who developed PSP. According to the knowledge of the authors, this report of PSP in a recreational athlete is the first of its kind described in South Africa. Owing to the possibility of life-threatening complications, it is important for sports physicians to be familiar with the important points in the history and to be made aware of the predisposing factors that may lead to PSP.
\end{abstract}

S Afr J Sports Med 2015;27(1):23-24. DOI:10.7196/SAJSM.603

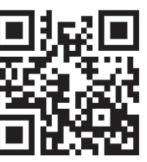

Pneumothorax is defined as the presence of gas in the pleural cavity. ${ }^{[1]}$ This can occur spontaneously or subsequent to direct trauma to the chest wall. Primary spontaneous pneumothorax (PSP) occurs in the absence of any underlying lung pathology, ${ }^{[1-3]}$ and although it is a well-recognised and well-described cause of acuteonset chest pain, the prevalence of this condition in the athletic community is uncommon. This may be owing to the low incidence of this condition in athletes, underreporting of cases and possibly missed diagnoses.

Tension pneumothorax is an extremely rare but life-threatening complication of PSP, and if suspected, should be treated as a medical emergency. ${ }^{[2]}$ This case report describes a rare occurrence of PSP in a recreational athlete and focuses on the athlete's medical history as well as the epidemiology and predisposing factors that may lead to PSP.

\section{Case report}

This case involves a 34-year-old male who worked as an engineer at a steel production plant. He participated in a number of indoor and outdoor sports. In his medical history, it was noted that he suffered from gastro-oesophageal reflux disease (GERD) secondary to a hiatus hernia, which was diagnosed in 2009. No further medical problems were reported. No known allergies were reported and he did not smoke. He did, however, have a positive family history, with one female sibling having developed PSP in recent years.

Six weeks prior to the onset of his symptoms, he competed in a high-altitude ultra-marathon trail run in cold and wet conditions. On the day before his symptoms started, he was playing action cricket. He did not experience any symptoms and or sustain any direct trauma to the chest wall during the match. He presented to his general practitioner (GP) with complaints of acute-onset chest pain on the right side, which woke him during the night. The pain progressively worsened the following day and did not respond to analgesic or antacid treatment. He did not complain of any shortness of breath, and attributed the pain to acid reflux.

The examination was noted to be unremarkable, with normal lung and heart sounds and normal vital signs. His GP agreed that the pain might be due to GERD. Antireflux treatment was prescribed and he was sent home. The pain gradually subsided over the following 5 days.

After 2 weeks of rest, he played another match of action cricket, but had to withdraw from the match because of chest pain and shortness of breath. A follow-up appointment with his GP was scheduled 3 weeks after the initial appointment.

Immediate chest radiographs were ordered, which showed a large pneumothorax with $60 \%$ of the pleural space occupied by air on the right side of the chest (Fig. 1). No mediastinal shift was noted on the

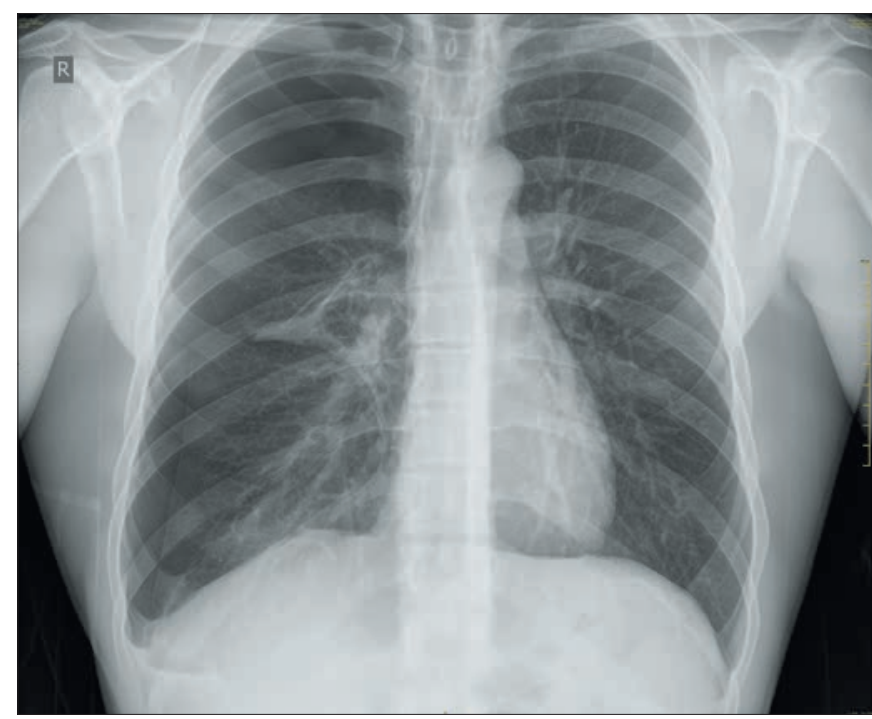

Fig. 1. X-ray of lungs clearly demonstrating pneumothorax on the right. 
radiograph. He was urgently referred to a cardiothoracic surgeon and was taken to the operating theatre the following day for a diagnostic bronchoscopy. The cause of the pneumothorax was found to be a ruptured bulla in the apex of the right lung. The surgeon continued to do a right posterolateral minithoracotomy, drained the organising pleural effusion, and performed a bullectomy and complete parietal pleurectomy of the right lung.

The pleura of the right lung were sent to the laboratory for histology, including Ziehl-Neelson staining to exclude tuberculosis infection and periodic acid-Schiff (PAS) staining to exclude fungal infection. Histology showed an eosinophylic pleuritis with reactive mesothelial hyperplasia. The Ziehl-Neelson stain and PAS stain both came back negative. He was discharged from hospital a few days later and his recovery since then has been uneventful.

\section{Discussion}

Pneumothoraces can broadly be categorised as either traumatic or spontaneous. Spontaneous pneumothorax can further be subdivided into two groups: primary spontaneous pneumothorax (PSP), where there is no history of underlying lung pathology; and secondary spontaneous pneumothorax (SSP), where there is pre-existing lung pathology. ${ }^{[1-3]}$

PSP is more common in males, with an incidence of 7.4 18 cases/100 000/year in males, and 1.2 - 6 cases/100 000/year in females. ${ }^{[2,3]}$ Other risk factors include smoking and being a tall, thin male between the ages of 10 and 30 years. ${ }^{[3]}$ Almost $10 \%$ of cases have a positive family history of PSP, which is linked to a specific gene mutation, folliculin, associated with a rare disease called Birt-HoggDubé syndrome. ${ }^{[3]}$ Changes in climate, with decreased atmospheric pressures and colder temperatures, have been identified as possible contributing factors in PSP. ${ }^{[4]}$

The most common presenting symptoms of PSP are acute-onset pleuritic chest pain and shortness of breath, which may improve over a period of 24 hours. ${ }^{[3]}$ Chest pain is an important and reliable symptom that is present in $80-95 \%$ of cases. ${ }^{[5]}$ The physical examination may reveal a decrease in breath sounds on the affected side, with hyper-resonance on percussion, crepitus on palpation and tracheal shift. ${ }^{[6]}$ These signs may be very difficult to elicit in a small pneumothorax, and a physician should have a high index of suspicion from the history.

The threshold for ordering chest radiographs should be very low if a possible pneumothorax is suspected. The majority of cases occur during rest, with $<10 \%$ of spontaneous pneumothoraces occurring during exercise. The major cause of PSP seems to be the rupture of bullae in the apices of one or both lungs. The exact cause of these bullae still remains unclear and warrants further investigation. ${ }^{[2,3]}$

PSP seems to be a rare condition among the athlete population. In this case report, it is evident that the athlete had a number of risk factors, including sex, age, exposure to cold weather conditions and decreased atmospheric pressure, as well as a positive family history, all of which need to be taken into account when an athlete presents with acute-onset chest pain. The recording of cases of PSP in the athletic community specifically is encouraged to help guide sports physicians to better diagnose and manage this condition. The research and development of safe return-to-play guidelines for these athletes are also recommended.

\section{References}

1. Braunwald E, Fauci AS, Kasper DL, eds. Harrison's principles of internal medicine. 15th ed. New York: McGraw-Hill, 2001.

2. Noppen M. Spontaneous pneumothorax: Epidemiology, pathophysiology and cause. Eur Respir Rev 2010;19(117):217-219. [http://dx.doi.org/10.1183/09059180.00005310]

3. Luh S. Diagnosis and treatment of primary spontaneous pneumothorax. J Zhejiang Univ Sci B 2010;11(10):735-744. [http://dx.doi.org10.1631/jzus.B1000131]

4. Haga T, Kurihara $M$, Kataoka $H$, Ebana $\mathrm{H}$. Influence of weather conditions on the onset of primary spontaneous pneumothorax: Positive association with decreased atmospheric pressure. Ann Thorac Cardiovasc Surg 2013;19(3):212-215. [http:// dx.doi.org/10.5761/atcs.oa.12.01884]

5. Sik EC, Batt ME, Heslop LM. Atypical chest pain in athletes. Curr Sports Med Rep 2009;8(2):52-58. [http://dx.doi.org/10.1249/JSR.0b013e31819c7d01]

6. Mensinger JM. Pneumothorax in a recreational athlete. Int J Athl Ther Train 2013;18(6):27-31.

\section{SAJSM CPD}

The CPD programme for SAJSM is being administered by Medical Practice Consulting.

CPD questionnaires must be completed online at www.mpconsulting.co.za

A maximum of 3 CEUs will be awarded per correctly completed test.

\section{INSTRUCTIONS \\ 1. The SAJSM March issue questionnaire can be found online at www.sajsm.org.za \\ 1. Read the journal. All the answers will be found there. \\ 2. Go to www.mpconsulting.co.za to answer the questions.}

Accreditation number: MDB015/166/02/2015 (Clinical) 